\title{
INHIBITION OF VOID DISSOLUTION BY VACANCIES
}

\author{
Y. Estrin ${ }^{1}$, G. Gottstein ${ }^{2}$ and L.S. Shvindlerman ${ }^{2,3}$ \\ ${ }^{1}$ Department of Mechanical and Materials Engineering, The University of Western Australia, \\ Nedlands WA 6907, Australia ${ }^{2}$ Institut für Metallkunde und Metallphysik, RWTH Aachen, \\ Kopernikusstrasse 14, D-52074 Aachen, Germany ${ }^{3}$ Institute of Solid State Physics, Russian \\ Academy of Sciences, Chernogolovka, Moscow District 142432, Russia
}

(Received March 9, 1999)

(Accepted March 15, 1999)

\begin{abstract}
Introduction
Dissolution of voids is a classical problem in the theory of sintering. The foundations of the theory were established by Frenkel, Pines and Geguzin (see Refs. 1 and 2 for a review). However, an important aspect of the problem appears to have been overlooked by previous workers. In a recent paper, the present authors identified a mechanism which inhibits processes associated with vacancy generation during grain growth, especially in fine grain sized materials [3]. The essence of the mechanism is the rise of the free energy of the system due to vacancy production in the course of defect 'dissolution' (decrease of the total area of grain boundaries) which under certain conditions may inhibit the rate of the primary process. A similar effect may also be operative in the case of void dissolution which is accompanied by a massive release of vacancies into the surrounding bulk of the material. In this paper we consider the conditions for intermittent temporary arrest of the void dissolution process leading to an effective decrease of the rate of dissolution.
\end{abstract}

\section{The Model}

We consider an ensemble of identical voids of radius $\mathrm{R}$ in an otherwise uniform solid. The number of voids per unit volume, $\mathrm{n}$, is assumed not to change during the process. The void size $\mathrm{R}$ and $\mathrm{n}$ are related to the volume $\mathrm{f}$ of voids (porosity) through

$$
f=\frac{4 \pi R^{3}}{3} n
$$

Introducing the surface energy per unit area, $\sigma$, one can express the Gibbs free energy of the system of voids and vacancies, $\mathrm{G}$, as [3]

$$
G=4 \pi R^{2} \sigma n+G\left(c^{e q}\right)+\frac{1}{2} \frac{N k T}{c^{e q}}\left(c-c^{e q}\right)^{2} .
$$

In eq. (2), $G$ is given per unit volume; the use of a series expansion of the Gibbs free energy of the vacancy sub-system restricted to the quadratic term in $\mathrm{c}-\mathrm{c}^{\mathrm{eq}}$ implies that the vacancy sub-system is not 


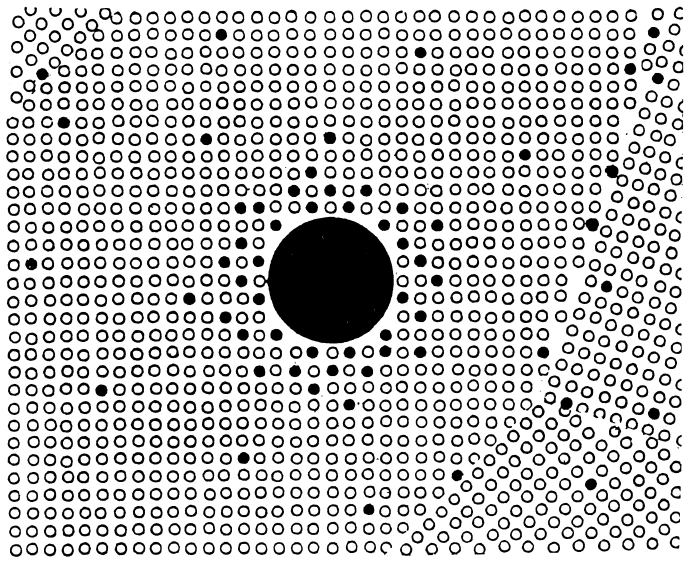

Figure 1. A schematic picture of a void with a vacancy atmosphere (black dots). The grain boundaries shown represent one possible type of sinks.

too far from equilibrium [3]. Here $\mathrm{c}^{\mathrm{eq}}$ is the thermal equilibrium vacancy concentration, $\mathrm{N}$ is the number of atomic sites per unit volume, $\mathrm{k}$ is the Boltzmann factor and $\mathrm{T}$ the absolute temperature.

To check the thermodynamic feasibility of decerease of void radius, we investigate the sign of the derivative of $\mathrm{G}$ with respect to time $\mathrm{t}$ which reads:

$$
\dot{G}=8 \pi R \sigma n \dot{R}+\frac{N k T}{c^{e q}}\left(c-c^{e q}\right) \dot{c}
$$

In order to consider the rate of variation with time of the radius of an individual void, $\dot{R}$, we write for the vacancy flux into the bulk:

$$
j=-D_{\nu} N \frac{c_{S}-\bar{c}}{R}
$$

Here $\mathrm{D}_{v}$ is the vacancy diffusivity in the bulk; $\mathrm{c}_{\mathrm{s}}$ and $\overline{\mathrm{c}}$ are the vacancy concentrations in close proximity of the void surface and in the bulk far away from it, respectively. It is assumed that the voids are not located at such vacancy sinks as dislocations or cell, subgrain or grain boundaries. An individual void and its vacancy atmosphere are sketched in Fig. 1.

The rate of change of the void radius follows from the balance of volume lost by the void and transferred to the bulk by the vacancies produced:

$$
-D_{\nu} N \Omega \frac{c_{S}-\bar{c}}{R} 4 \pi R^{2}=4 \pi R^{2} \dot{R}
$$

or

$$
\dot{R}=-D_{\nu} N \Omega \frac{c_{S}-\bar{c}}{R} .
$$

Here $\Omega$ denotes the volume of a vacancy.

To calculate the vacancy concentration in the bulk, $\bar{c}$, we introduce the radius of the "catchment region' $\mathrm{L}$ of an individual void which is defined by 


$$
\frac{4 \pi}{3} L^{3} n=1
$$

As $\mathrm{L} \gg \mathrm{R}$, the concentration $\bar{c}$ can be assumed to prevail in practically the entire region of radius $\mathrm{L}$. The variation of $\bar{c}$ with time can be considered to result from a competition between vacancy 'injection' from the void and losses to bulk sinks. The rate of increase of the vacancy content in the bulk due to vacancy injection, $\dot{\bar{c}}^{+}$, thus follows from

$$
\frac{4 \pi}{3} L^{3} \dot{\bar{c}}^{+}=4 \pi R^{2} D_{\nu} \frac{c_{S}-\bar{c}}{R}
$$

which, using eq. (7), yields

$$
\dot{\bar{c}}^{+}=4 \pi R n D_{\nu}\left(c_{S}-\bar{c}\right)
$$

The rate of decrease of $\bar{c}$ due to vacancy annihilation at sinks (e.g. edge dislocations or grain boundaries) is given by

$$
\dot{\bar{c}}^{-}=-\frac{D_{v}}{d^{2}} \bar{c}
$$

where d denotes the sink spacing. The sinks may be associated with dislocations, cell, subgrain or grain boundaries. The sinks are assumed to be unsaturable and the vacancy concentration at sinks is taken to be equal to zero. Finally, the equation for the time derivative of $\bar{c}$ reads

$$
\dot{\bar{c}}=4 \pi R n D_{v}\left(c_{S}-\bar{c}\right)-\frac{D_{v}}{d^{2}} \bar{c}
$$

Our aim is now to solve the set of coupled equations (6) and (11) and to assess the sign of the time derivative of $\mathrm{G}$,

$$
\dot{G}=8 \pi R \sigma n \dot{R}+\frac{N k T}{c^{e q}}\left(\bar{c}-c^{e q}\right) \dot{\bar{c}}
$$

cf. eq. (3). The vacancy concentration at the void surface, $c_{S}$, is given by the Gibbs-Thomson equation

$$
c_{S}=c^{e q} \exp \left(\frac{2 \Omega \sigma}{k T R}\right) \text {. }
$$

\section{Arrest of Void Dissolution}

Some preliminary conclusions can already be made if the time derivatives of $\mathrm{R}$ and $\bar{c}$ with respect to time are substituted from eqs. (6) and (11) into eq. (12). This yields

$$
\dot{G}=4 \pi N n D_{\nu} R k T\left(c_{S}-\bar{c}\right)\left\{-\frac{2 \Omega \sigma}{k T R}+\frac{\bar{c}-c^{e q}}{c^{e q}}\left[1-\frac{1}{4 \pi n d^{2} R} \cdot \frac{\bar{c}}{c_{S}-\bar{c}}\right]\right\}
$$

Equation (14) is a key to the understanding of the inhibiting effect of vacancies in the void dissolution. At the very beginning of the process, as long as $\bar{c}$ is still close to $c^{e q}$, the sign of $\dot{G}$ is determined by the first term in the curly brackets, i.e. it is negative and, as could be expected, void dissolution is promoted thermodynamically. However, with the growth of $\bar{c}$ due to vacancy emission, the second term in the curly brackets gains on importance and, for sufficiently large $\mathrm{d}$ when the vacancy removal to 
sinks is not efficient, the expression in the curly brackets may become positive. The dissolution of voids will be suppressed when this condition is reached.

To investigate this behaviour in more detail, we solve the set of differential equations for $R$ and $\bar{c}$, eqs. (6) and (11). Combining eqs. (6) and (11) into

$$
\frac{d \bar{c}}{d R}=-4 \pi \frac{R^{2}}{\Omega}
$$

yields upon integration

$$
\bar{c}=c^{e q}=\frac{4 \pi}{3}\left(R_{o}^{3}-R^{3}\right) \frac{n}{N \Omega} .
$$

It can easily be seen that eq. (16) is an expression of the fact that the entire volume lost by the voids is accommodated by the vacancies in the bulk. Now, using a series expansion of $\mathrm{R}$ near its initial value $\mathrm{R}_{\mathrm{o}}$ in eq. (11) and solving the resulting differential equation in $\bar{c}$ we obtain

$$
\bar{c}=c^{e q} \exp (-t / \hat{t})+c^{\infty}[1-\exp (-t / \hat{t})]
$$

where

$$
c^{\infty}=\frac{4 \pi n D_{\nu} R_{o}}{4 \pi n D_{\nu} R_{o}+D_{\nu} / d^{2}}\left(1+\frac{2 \Omega \sigma}{k T R_{o}}\right) c^{e q}
$$

and

$$
\frac{1}{\hat{t}}=4 \pi n D_{\nu} R_{o}+\frac{D_{v}}{d^{2}}
$$

Strictly speaking, eq. (17) should only be considered for sufficiently small times for which linearisation with respect to the difference $\mathrm{R}-\mathrm{R}_{\mathrm{o}}$ used in the derivation is admissible. The validity of this assumption will be checked below. It should be noted that in deriving eq. (17) the quantity $2 \Omega \sigma / k T R_{o}$ was assumed to be small compared to unity. For typical parameter values and the temperature around $1000 \mathrm{~K}$, the latter condition is fulfilled for R larger than $10^{-6} \mathrm{~cm}$.

As the quantity $c_{s}-\bar{c}$ is always positive, the sign of $\dot{G}$ is determined by the expression in the curly brackets which reads

$$
\Psi=-\frac{2 \Omega \sigma}{k T R}+\frac{\bar{c}-c^{e q}}{c^{e q}}\left[1-\frac{1}{4 \pi n d^{2} R} \cdot \frac{\bar{c}}{c_{S}-\bar{c}}\right] \cong-\frac{2 \Omega \sigma}{k T R_{o}}+\frac{D_{v}}{d^{2}} \frac{(\chi-1)^{2}}{\chi} \cdot t
$$

where

$$
\chi=4 \pi n d^{2} \frac{2 \Omega \sigma}{k T}
$$

Obviously, at very small times, $\Psi$ is negative, and so is $\dot{G}$. However, after a time

$$
t^{*}=\frac{2 \Omega \sigma}{k T R_{o}} \cdot \frac{\chi}{(\chi-1)^{2}} \frac{d^{2}}{D_{\nu}}
$$

the void dissolution will be arrested. Only for $\chi$ tending to unity will the time to locking, $\mathrm{t}^{*}$, be tending to infinity. 
Now we should prove that two conditions used in the above derivation are fulfilled up to the time $t^{*}$. These are:

$$
\text { (i) }\left|R\left(t^{*}\right)-R_{o}\right| / R_{o}=\frac{\bar{c}\left(t^{*}\right)-c^{e q}}{4 \pi R_{o}^{2}} \frac{N \Omega}{n} \ll 1
$$

and

$$
\text { (ii) } \frac{t^{*}}{\hat{t}} \ll 1
$$

Using eq. (17) linearised with respect to time, as well as eqs. (18), (19), (21) and (22), these inequalities assume the form

$$
\frac{2 \Omega \sigma}{k T R_{o}} \cdot \frac{N \Omega c^{e q}}{4 \pi R_{o}^{3} n} \frac{\chi}{(\chi-1)^{2}} \ll 1
$$

and

$$
2 \chi \ll 1
$$

Inequality (25) is fulfilled for all $\chi$ not too close to unity, particularly, for $\chi$ much smaller than $1 / 2$ as required by the second condition, i.e. inequality (26). Hence, the validity of the expression for $t^{*}$, eq. (22), is warranted for $\chi \ll 1 / 2$. For most physically relevant situations the latter condition is fulfilled, at least in the inirial stages of the process. Indeed, rewriting $\chi$ as $\chi=\left(f_{o} / 3\right)\left(d / R_{o}\right)^{2}\left(2 \Omega \sigma /\left(k T R_{o}\right)\right)$, where $f_{o}$ is the initial volume fraction of voids, one can see that for $f_{o}$ of the order of percent and $R_{o}$ of the order of $10^{-6} \mathrm{~cm}$ or larger, this condition is safely fulfilled for $\mathrm{d}$ of the order of $10^{-5} \mathrm{~cm}$. This sink spacing compares favourably with a typical cell size or the average dislocation spacing.

A typical time for vacancy removal after locking is given by $d^{2} / D_{v}$, cf. eq. (11). After this time, void dissolution will re-start leading to renewed injection of vacancies into the surrounding bulk. Repeated locking and unlocking of the process will ensue. The system is 'open' for the void dissolution process to occur within the time $\mathrm{t}^{*}$, that is for a small fraction of $d^{2} / D_{v}$, cf. eq. (22), and 'locked' for a time of the order of $d^{2} / D_{v}$.

\section{Discussion and Conclusions}

The effect of vacancies generated during void dissolution on the behaviour of the 'parental' voids was considered. It was shown that the contribution of vacancies injected into the bulk to the Gibbs free energy must have an inhibiting effect on the void dissolution. This inhibition is of a cyclic nature. After a time $t^{*}$, the process of void dissolution gets suppressed by the vacancies. It takes the system a much larger time to remove the vacancies to the sinks. It is interesting to note that the time $t^{*}$ during which the void dissolution process is active increases with each cycle as the void radius becomes smaller and smaller.

For simplicity, an ensemble of identical voids was considered. However, the essence of the phenomenon is captured by the simplified model. In an ensemble of voids with a size distribution, the void activity time $t^{*}$ between two successive arrests of dissolution will be different for voids of different radius. According to eq. (22), the activity time of small voids will be larger than that of bigger ones. This will change the evolution kinetics of an ensemble of voids and the void size distribution. It would be of interest to re-consider the Lifshitz-Slyozov-Wagner kinetics of an ensemble of voids in this light. 
One obvious area where the effect considered may be of particular interest is sintering where the reduction of porosity is a problem of paramount importance.

\section{Acknowledgments}

Support from the Deutsche Forschungsgemeinschaft (DFG Grant 438 113/130/0) and from the International Office of the BMBF (Project AUS-032) is gratefully acknowleged. One of the authors (YE) would like to thank the Alexander von Humboldt Foundation for generous support through a Humboldt Research Award.

\section{References}

1. E. Geguzin, Physik des Sinterns, VEB Deutscher Verlag für Grundstoffindustrie, Leipzig (1973).

2. H. E. Exner and E. Arzt, in Physical Metallurgy, ed. R. W. Cahn and P. Haasen, p. 2627, North- Holland, Amsterdam (1996).

3. Y. Estrin, G. Gottstein, and L. S. Shvindlerman, Acta Mater. submitted. 